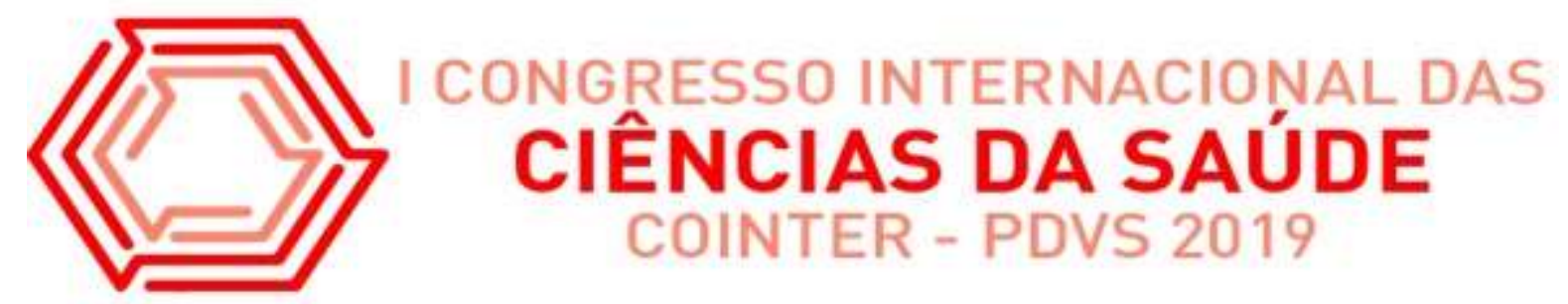

\title{
VIOLÊNCIA AUTOPROVOCADA EM ADOLESCENTES NO PERÍODO DE 2013- 2017: UM GRAVE PROBLEMA DE SAÚDE PÚBLICA EM PERNAMBUCO
}

\author{
VIOLENCIA AUTOCUPADA EN ADOLESCENTES 2013-2017: UN GRAVE \\ PROBLEMA DE SALUD PÚBLICA EN PERNAMBUCO
}

\section{SELF-ADVOCATED VIOLENCE IN ADOLESCENTS IN THE 2013-2017 PERIOD: A SERIOUS PUBLIC HEALTH PROBLEM IN PERNAMBUCO}

\author{
Apresentação: Comunicação Oral \\ Laís Eduarda Silva de Arruda ${ }^{1}$; Luís Roberto da Silva ${ }^{2}$; Jonathan Willams do Nascimento ${ }^{3}$ \\ Thiago da Silva Freitas ${ }^{4}$; Emília Carolle Azevedo de Oliveira ${ }^{5}$
}

DOI: $\underline{\text { https://doi.org/10.31692/ICOINTERPDVS.2019.0013 }}$

\begin{abstract}
Resumo
As violências auto provocadas são um grave problema de saúde pública com repercussões diversas na sociedade, possui causas variadas que vão desde fatores biológicos a socioculturais, que têm aumentado nos últimos anos. São definidas como qualquer comportamento intencional envolvendo agressão direta ao corpo, sem intenção consciente de suicídio, no entanto, podendo culminar neste desfecho negativo. Visto isso, esta produção tomou como objetivou analisar os casos de lesões auto provocadas no estado de Pernambuco no período de 2013 a 2017 e discutir sobre este problema de saúde pública. Para isto, utilizou-se exclusivamente dados secundários, obtidos através do Sistema Informação de Agravos de Notificação (SINAN), referentes a frequência de lesões auto provocadas por adolescentes em Pernambuco, entre os anos de 20132017, em seguida estes foram organizados em planilhas do Excel 2016® para elaboração da análise e criação de gráficos e tabelas. Dentre os principais resultados, obteve-se que o número de lesões auto provocadas por adolescentes têm aumentado nos últimos anos e foi evidenciado que o silêncio da sociedade em relação ao tema, têm colaborado para este aumento, pois isto

\footnotetext{
${ }^{1}$ Graduanda do curso de Bacharelado em Saúde Coletiva, Universidade Federal de Pernambuco - Centro Acadêmico de Vitória, laisarruda63@gmail.com

${ }^{2}$ Graduando do curso de Bacharelado em Saúde Coletiva, Universidade Federal de Pernambuco - Centro Acadêmico de Vitória, robertosillva059@gmail.com

${ }^{3}$ Graduando do curso de Bacharelado em Saúde Coletiva, Universidade Federal de Pernambuco - Centro Acadêmico de Vitória, jonathannascimento 874@outlook.com

${ }^{4}$ Biólogo licenciado pela Universidade de Pernambuco. Especialista em Saúde pública e Ensino da BiologiaFACOL. Mestrando em Neurociências-UFPE, thiago-25@hotmail.com

${ }^{5}$ Doutoranda em Saúde Pública do Instituto Aggeu Magalhães/Fiocruz/PE - Docente do Curso de Saúde Coletiva da Universidade Federal de Pernambuco, emiliacarolle@hotmail.com
} 
não é discutido e a sociedade, em geral, falha ao não notar ou ignorar estes sinais de sofrimentos de adolescentes e camuflando assim, esta problemática crescente na saúde pública, a falta de informação e explanação sobre os riscos dos comportamentos autodestrutivos também contribui para este agravamento. Foi identificado também, que a maioria dos casos registrados foram em adolescentes do sexo feminino em todo o período estudado constatando o sofrimento deste público, que pode estar relacionado com as questões de gênero e a herança machista que ainda persiste na sociedade brasileira, da submissão da mulher em relação ao homem. Com relação a raça/cor, notou-se uma predominância em pessoas pardas, seguida das brancas, entretanto ressalta-se a alta proporção $(14,07 \%)$ de fichas com esse quesito ignorado, o que pode resultar em equívocos de interpretação. Visto isso, é urgente a necessidade de fortalecer as ações e programas voltadas a prevenção deste problema através da criação e efetivação de políticas públicas. Também é preciso capacitar os profissionais de saúde e àqueles que trabalham com adolescentes para que estes possam identificar indícios destas práticas e seja possível evitar o agravamento destas. Além da extrema importância de se colocar este assunto em pauta na sociedade e quebrar o com estigmas e o tabu criados socialmente sobre as autolesões e suicídio.

Palavras-Chave: Violência, Comportamento do Adolescente, Saúde Pública.

\section{Resumen}

La violencia autoinfligida es un grave problema de salud pública con diversas repercusiones en la sociedad y tiene diversas causas, desde factores biológicos hasta socioculturales, que han aumentado en los últimos años. Sin embargo, se definen como cualquier comportamiento intencional que implique agresión directa al cuerpo, sin intención consciente de suicidio, que pueda culminar en este resultado negativo. Ante esto, esta producción tuvo como objetivo analizar los casos de lesiones autoinfligidas en el estado de Pernambuco de 2013 a 2017 y debatir sobre este problema de salud pública. Para este propósito, solo se usaron datos secundarios, obtenidos a través del Sistema de Información de Enfermedades de Notificación (SINAN), sobre la frecuencia de autolesiones causadas por adolescentes en Pernambuco entre 2013-2017, y luego se organizaron en hojas de cálculo. de Excel 2016® para análisis y gráficos. Entre los principales resultados, se encontró que el número de autolesiones causadas por los adolescentes ha aumentado en los últimos años y se evidenció que el silencio de la sociedad en relación con el tema, ha contribuido a este aumento, porque esto no se discute y la sociedad, En general, no se da cuenta o ignora estos signos de sufrimiento adolescente y, por lo tanto, camuflando este creciente problema de salud pública, la falta de información y explicación sobre los riesgos del comportamiento autodestructivo también contribuye a este agravamiento. También se identificó que la mayoría de los casos reportados fueron en mujeres adolescentes durante todo el período de estudio, señalando el sufrimiento de este público, que puede estar relacionado con cuestiones de género y la herencia chovinista que aún persiste en la sociedad brasileña, de la sumisión de las mujeres. Mujer relativa al hombre. Con respecto a la raza / color, hubo un predominio en las personas marrones, seguido del blanco, sin embargo, se destaca la alta proporción (14.07\%) de chips con este aspecto ignorado, lo que puede dar lugar a una mala interpretación. Ante esto, existe una necesidad urgente de fortalecer las acciones y los programas destinados a prevenir este problema mediante la creación e implementación de políticas públicas. También es necesario capacitar a profesionales de la salud y a quienes trabajan con adolescentes para que puedan identificar signos de estas prácticas y prevenir su agravamiento. Además de la extrema importancia de poner este tema en la agenda de la sociedad y romper el estigma y el tabú socialmente creado sobre la autolesión y el suicidio. 
Palabras Clave: Violencia, Conducta del Adolescente, Salud Pública.

\begin{abstract}
Self-inflicted violence is a serious public health problem with diverse repercussions on society. It has varied causes ranging from biological to sociocultural factors, which have increased in recent years. They are defined as any intentional behavior involving direct aggression to the body, without conscious intention of suicide, however, which may culminate in this negative outcome. Given this, this production aimed to analyze the cases of self-inflicted injuries in the state of Pernambuco from 2013 to 2017 and discuss about this public health problem. For this purpose, only secondary data, obtained through the Notification Disease Information System (SINAN), concerning the frequency of self-harm caused by adolescents in Pernambuco between 2013-2017, were used and then organized into spreadsheets. of Excel 2016® for analysis and graphing. Among the main results, it was found that the number of self-inflicted injuries by adolescents has increased in recent years and it was evidenced that the silence of society in relation to the theme, have contributed to this increase, because this is not discussed and society, In general, it fails to notice or ignore these signs of adolescent suffering and thus camouflaging this growing public health problem, the lack of information and explanation about the risks of self-destructive behavior also contributes to this aggravation. It was also identified that most of the reported cases were in female adolescents throughout the study period, noting the suffering of this public, which may be related to gender issues and the chauvinistic inheritance that still persists in Brazilian society, of the submission of women. Woman relative to man. Regarding race / color, there was a predominance in brown people, followed by white, however it is emphasized the high proportion $(14.07 \%)$ of chips with this item ignored, which may result in misinterpretation. Given this, there is an urgent need to strengthen actions and programs aimed at preventing this problem through the creation and implementation of public policies. It is also necessary to train health professionals and those who work with adolescents so that they can identify signs of these practices and to prevent their aggravation. In addition to the extreme importance of putting this issue on the agenda in society and breaking the socially created stigma and taboo on self-harm and suicide.
\end{abstract}

Keywords: Violence, Adolescent Behavior, Public Health.

\title{
Introdução
}

O suicídio é considerado um dos mais antigos temas relacionados à saúde dos indivíduos e à forma como são afetados pelas sociedades e coletividades nas quais vivem, sendo definido como um ato intencional para acabar com a própria vida (RIBEIRO, MOREIRA, 2018).

Segundo a Organização Mundial de Saúde (OMS), em 2012 ocorreram 804 mil óbitos por suicídio no mundo com uma taxa de, aproximadamente, 11,4 para cada 100.000 habitantes. Diante disso, em 2013, durante a $66^{\mathrm{a}}$ assembleia mundial de saúde da OMS, foi adotada a meta de se reduzir 10\% das taxas de suicídio nos países até 2020 (AGUIAR \& CARVALHO, 2018). 
Comportamentos suicidas não fatais apresentam-se sob a forma de ideação suicida, quando há pensamentos que instigam o desejo de dar fim à vida e se agrava quando acompanhados de um plano suicida sobre o método de auto aniquilamento. A tentativa de suicídio envolve condutas voltadas para se ferir em que há intenção de se matar, podendo ocasionar ferimento ou morte. Quando a tentativa de suicídio resulta em morte, passa a ser definida como suicídio. Todavia, o comportamento suicida se refere a um tipo de conduta da pessoa que busca se ferir ou se matar (OMS, 2002; OMS, 2014).

A lesão autoprovocada é a violência que a pessoa inflige a si mesmo, sendo subdividida em comportamento suicida e em autoagressão (envolve eventos de automutilação, incluindo desde as formas mais leves, como arranhaduras, cortes e mordidas até as mais severas, como amputação de membros). A autolesão representa qualquer comportamento intencional envolvendo agressão direta ao corpo, sem intenção consciente de suicídio (GIUSTI, 2013). Os comportamentos auto lesivos mais comuns são cortes superficiais na pele, arranhões, mordidas, queimaduras, bater uma parte da estrutura física contra a parede e enfiar objetos pontiagudos no corpo (CEDARO, NASCIMENTO, 2013).

Klonsky et al. (2015) afirmam existir uma alta incidência de caso de autolesão em adolescentes, seja na população em geral quanto nas amostras psiquiátricas, embora não seja um fenômeno novo, há escassa oferta de tratamentos comprovados. O mapa de violência de Waiselfisz (2019), afirma que o número de casos de lesões autoprovocadas aumentou no Brasil de forma significativa. Dados estatísticos mostram o crescimento do número de casos nas décadas de 1980, 1990 e em 2012, com taxas de 2,7\%, 18,8\% e 33,3\%, respectivamente.

No intervalo entre 2002 e 2012, observou-se um total de suicídios no Brasil que passou de 7.726 para 10.321 , o que evidenciou um aumento de $33,6 \%$ nesse período. Quando comparados ao crescimento populacional do país, nesse mesmo período, o aumento do número de suicídio foi maior, correspondendo à $11,1 \%$, excedendo em larga escala os homicídios e a mortalidade nos acidentes de transporte que obtiveram taxas de crescimento de $2,1 \%$ e $24,5 \%$, respectivamente (OMS, 2003).

O suicídio é um fenômeno muito complexo que impacta tanto a dimensão individual quanto coletiva e que ocorre pela convergência de diversos fatores de risco, dentre esses genéticos, psicológicos, sociais e culturais combinados com experiências de perda e traumas (CICOGNA, HILLESHEIM \& HALLAL, 2019).

Mesmo que se tenha indícios na literatura que demonstrem a frequência de autolesão 
entre adolescentes e do aumento nos últimos anos da prática desse comportamento (MUEHLENKAMP et al., 2012; PLENER et al., 2016), existe escassez de evidências publicadas com amostra de público adolescente no Brasil.

A adolescência é um dos períodos de desenvolvimento marcado por grandes transformações biológicas, psicológicas e sociais. Essas transformações aparecem acompanhadas de conflitos e angústias perante uma realidade de contradições e busca de identidade.

Assim, justifica-se a realização deste trabalho devido preocupação com o aumento dos casos de lesões autoprovocadas nos últimos anos. Dado isso, é importante conhecer como este processo tem se dado, seus impactos na saúde pública e essencialmente, devido a urgente necessidade de se divulgar produções científicas sobre o tema para a sociedade a fim de que haja uma soma de forças na prevenção do suicídio.

Deste modo, este estudo objetivou analisar os casos de lesões autoprovocadas no estado de Pernambuco no período de 2013 a 2017 e discutir sobre este problema de saúde pública.

\section{Fundamentação Teórica}

A violência autoprovocada é um agravo de bastante importância para o setor saúde, uma vez que é uma das violências que acometem bastante uma relevante parcela da população e que termina se configurando no suicídio (BARBOSA, MACEDO, SILVEIRA, 2011). Segundo dados da Organização Mundial de Saúde (OMS, 2000), estima-se que em 2020, aproximadamente 1,53 milhão de pessoas no mundo morrerão por suicídio.

Os efeitos individuais e sociais do suicídio entre jovens no Brasil são analisados a partir dos casos registrados como lesões autoprovocadas intencionalmente. Deve ser lembrado que os problemas de falta de registros completos são importantes e vão além das lacunas típicas destas bases administrativas (RIBEIRO \& MOREIRA, 2018).

Ressalta-se que para cada caso de suicídio consumado existam entre 10 e 20 tentativas, o que reforça a tese da importância da detecção precoce dos riscos presentes nos casos dos deprimidos suicidas. Os dados oficiais relativos a tentativas de suicídio são mais falhos do que os de mortalidade por suicídio. As informações disponibilizadas pela OMS estão subestimadas, pois nem todos os países enviam seus dados de atualização (MELEIRO, 2004).

O suicídio fatal está entre as dez principais causas de óbitos e atinge principalmente jovens e adultos, com impactos sociais, econômicos, familiar, comunitário e na sociedade em 
geral. E apesar de ter ocorrido uma redução, cerca de 75\% dos óbitos auto infligidos ocorrem em países subdesenvolvidos e em desenvolvimento (BAHIA et al., 2017).

Estima-se que, por ano, cerca de 800.000 suicídios ocorrem no mundo e isto, representa um óbito a cada 40 segundo por esta causa. Na população jovem (15 a 29 anos) é tida como a segunda causa de mortalidade global, segundo a OMS (CICOGNA, HILLESHEIM \& HALLAL, 2019).

Para Bahia et al. (2017), os principais fatores de risco associados ao comportamento suicida são: problemas biológicos, médicos, ambientais, psiquiátricos, psicológicos, filosófico existenciais e por motivações sociais. Os fatores psiquiátricos e psicológicos mais comuns são: depressão, problemas relacionados ao estado de humor e afetivo bipolar; esquizofrenia; ansiedade e transtornos de personalidade; alcoolismo; desesperança e solidão e comorbidades.

Freud caracterizava a melancolia em termos psíquicos, por um abatimento doloroso, uma cessação do interesse pelo mundo exterior, perda da capacidade de amar, inibição de toda a atividade e principalmente a diminuição da autoestima que se expressa em auto recriminações e ofensas a própria pessoa e pode chegar a uma delirante expectativa de punição (FREUD, 1914-1916).

Todo o estigma que há entorno da temática, impede a procura de ajuda por partes das pessoas que estão em sofrimento e necessitam de ajuda e assim, evitar o óbito. Assim, é essencial falar sobre essa temática, pois, sabe-se que isso contribui efetivamente como fator de prevenção e pode, até contrapor as causas (BRASIL, 2017). Trata-se de um tema de extrema relevância para mobilização do poder público para construção e efetivação de políticas públicas voltadas a prevenção do suicídio sob uma minuciosa óptica (ALVES \& CADETE, 2015). Dado isto, é importante falar sobre o tema sem alarmismo, enfrentando-se toda estigmatização criada sobre o tema, com o intuito de conscientizar e estimular a prevenção, para que assim, se reduza o atual cenário crítico desta problemática (BRASIL, 2017).

Com relação a variável sexo, no Brasil, nota-se um cenário que vai assemelhar-se ao mundial, com maior incidência dos casos de lesões autoprovocadas no sexo feminino. Fato que deve ser destacado devido a vulnerabilidade feminina na sociedade, que carrega traços de uma cultura machista e as oprime. E por isto, tendem a mais tentativas de suicídio, enquanto homens têm mais êxito em acabar com a própria vida (BAHIA et al., 2017).

Existe um potencial importante do serviço de urgência e emergência em detectar e cuidar de casos em estágios ainda primários do fenômeno e de impedir um dano ainda mais 
grave para a vítima (BAHIA et. al, 2017). É recomendado que todos os pacientes que chegam nas emergências em situação que sugira associação com lesão autoprovocada sejam rastreados quanto ao risco de cometer suicídio. Uma intervenção breve, com o delineamento de um plano de segurança que coloque o paciente em contato com serviços de saúde mental é uma das ações mais benéficas e eficazes (BALLARD et al., 2014).

Discute-se ainda, sobre a qualidade do registro das informações, uma vez que, os valores culturais e sociais, podem impactar diretamente no procedimento e subestimar a real situação do problema no país e no mundo (CICOGNA, HILLESHEIM \& HALLAL, 2019). Com a fidedignidade do dado, possibilita-se estudar a distribuição dos casos de lesões autoprovocadas no país e conhecer o perfil das vítimas que são atendidas nos serviços de saúde, além de colaborar com a implantação de políticas públicas (AGUIAR \& CARVALHO, 2018).

Outro ponto que merece destaque, é a qualificação dos profissionais de saúde em identificarem casos de autolesões provocadas nos casos que atende e para além disso, seja capaz de acolher a pessoa em sofrimento que tentou tirar a própria vida com o intuito de amenizar e prevenir o desfecho negativo através da humanização do atendimento (MACHIN, 2007).

\section{Metodologia}

Foi realizado um estudo epidemiológico descritivo com abordagem quantitativa dos casos de lesões autoprovocadas por adolescentes notificadas no estado de Pernambuco, no período de 2013 a 2017, disponíveis no Sistema de Informação de Agravos de Notificação (SINAN). Por ser um estudo descritivo, caracteriza-se por determinar a distribuição de doenças ou condições relacionadas à saúde, segundo tempo, lugar e características dos indivíduos (ROUQUAYROL \& BARRETO, 2003, p.83).

Adotou-se como critério para definição da faixa etária de adolescentes, a classificação da Organização Mundial de Saúde (OMS), que define está sendo de 10-19 anos. Os dados foram processados no TabNet disponibilizado pelo departamento de informática do SUS (DATASUS) e importados para o TabWin Versão 4.15, para realização de algumas análises.

Além da faixa etária de adolescentes do estado, também foram selecionadas as variáveis de sexo e raça/cor para serem também analisadas e descritas neste trabalho, a partir das informações contidas no SINAN. Posteriormente, esses dados foram organizados em planilhas do Microsoft Office Excel 2016® para elaboração de gráficos e tabelas.

Além disso, com relação à aprovação do Comitê de Ética em Pesquisa (CEP), este 
estudo não precisou ser submetido e aprovado, uma vez que foram utilizados exclusivamente dados e informações de domínio público.

\section{Resultados e Discussão}

A partir das tabulações de dados referentes às lesões autoprovocadas no estado de Pernambuco (2013-2017), foi evidenciado um aumento de casos ao longo dos anos. Com registro total de 6559 , dos quais cerca de 27,09\% (1777) foi cometida por adolescentes, segundo informações contida no SINAN (Tabela 1).

Tabela 1- Lesões autoprovocadas por adolescentes no estado de Pernambuco nos anos de 20132017.

\begin{tabular}{lccc}
\hline Ano & & Total & \% \\
\hline $\mathbf{2 0 1 3}$ & 297 & 16,71 \\
\hline $\mathbf{2 0 1 4}$ & 277 & 15,58 \\
\hline $\mathbf{2 0 1 5}$ & 303 & 17,05 \\
\hline $\mathbf{2 0 1 6}$ & 334 & 18,8 \\
\hline $\mathbf{2 0 1 7}$ & 566 & 31,86 \\
\hline Total & 1777 & 100
\end{tabular}

Fonte: Sistema de Informações de Agravos de Notificação

Foram registrados 297, 277, 303, 334 e 566 casos nos anos de 2013, 2014, 2015, 2016 e 2017, respectivamente (Tabela 1). Em todos os anos estudados, houve um aumento das notificações destes acontecimentos, exceto em 2014, que registrou uma redução de 20 casos, quando comparado com o ano anterior (Gráfico 1). O ano de 2017 registrou um aumento de $13,06 \%$ casos a mais que no ano de 2016 e se destacou entre os demais anos estudados.

Gráfico 1- Evolução das lesões autoprovocadas em adolescentes em Pernambuco no período de 2013 a 2017. 


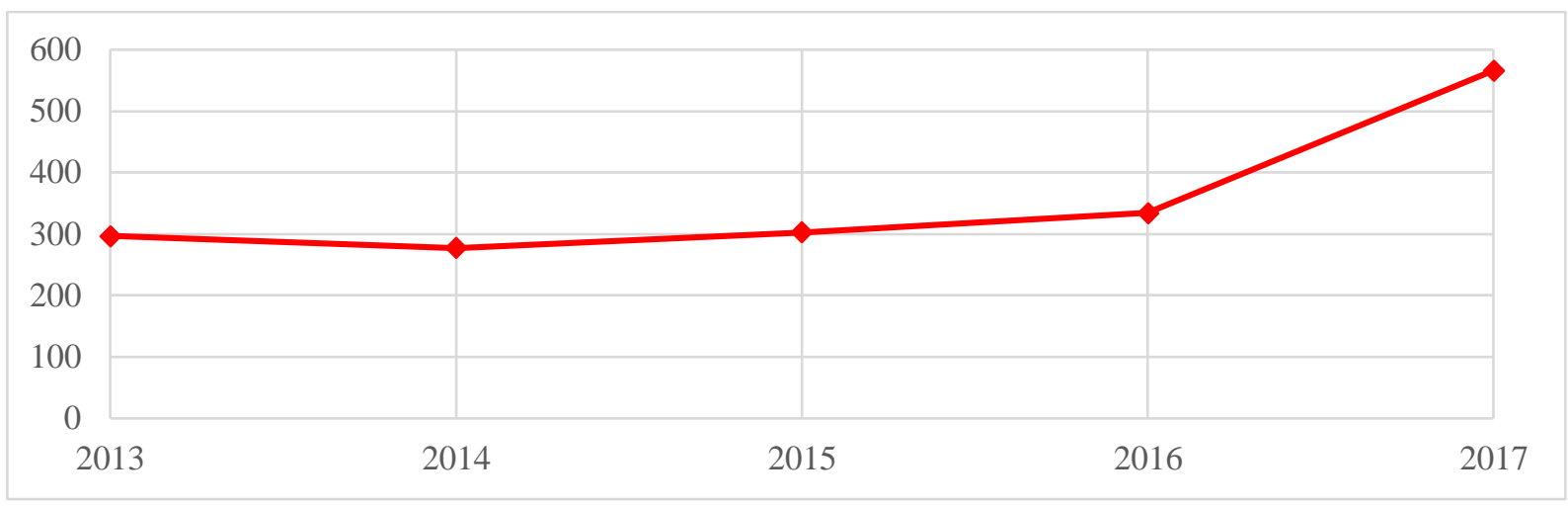

Fonte: Sistemas de Informações de Agravos de Notificação

Diante desse aspecto, pode-se notar que há um aumento bastante significativo das lesões autoprovocadas por adolescentes de 2016 a 2017, o que para Botega (2002) pode estar relacionado com o silêncio ao longo dos anos pela sociedade, autoridades responsáveis, profissionais de saúde e familiares, camuflando assim um grande problema de saúde pública no Estado. Assim, o fato é que esse silêncio só corrobora para que essas causas aumentem a cada ano e naturalizem a ideia das lesões autoprovocadas que consequentemente se dá ao suicídio.

Além disso, a falta de informação e a explanação sobre os riscos que os comportamentos autodestrutivos trazem para a vida do adolescente, por parte dos próprios familiares, bem como pelos próprios profissionais de saúde, fazem com que a ideação suicida comece a se desenvolver de forma mais rápida, pelo fato de não serem compreendidos em seus problemas, começando a se destinar pelas lesões autoprovocadas que começam de forma mínima e vão se perpetuando cada vez mais, até chegar ao ponto mais extremo, que é o suicídio.

Em relação ao sexo, foi identificado que cerca de 72,54\% (1289) das ocorrências registradas foram em adolescentes do sexo feminino (Tabela 2). A quantidade no sexo feminino foi sempre acima do sexo masculino em todos os anos estudados, correspondendo a mais de 50\%. Em 2013 correspondeu a 73,74\%, em 2014 (72,92\%), 2015 (67,33\%), 2016 (73,65\%) e em 2017 (73,85\%). Nesse último (2017), houve um aumento dos casos tanto no sexo masculino quanto no feminino, sendo esse maior que o dobro do sexo masculino (Gráfico 2).

Com isso, o aumento dos comportamentos autodestrutivos no sexo feminino é algo bastante alarmante, uma vez que se supera o sexo masculino em todos os anos $(2013,2014$, 2015, 2016, 2017), o que pode estar claramente relacionado aos aspectos de gênero incluídos 
na sociedade, no qual a mulher se encontra como um ser que deve submissão ao homem, sendo determinada pelo corpo social como inferior ao sexo masculino. Assim, também se percebe os processos de inclusão no mercado de trabalho, pautada na visão cultural machista da sociedade, dentro de uma masculinidade hegemônica efetivamente ativa (HESKETH \& CASTRO, 1978).

Com isso, há uma inferioridade do sexo feminino, fazendo com que o aumento das lesões seja maior na mulher que sofre uma maior opressão da sociedade. Para Monteiro et al. (2015), as lesões autoprovocadas são consideradas importante problema de saúde pública, pois constituem um sinalizador de mal-estar e sofrimento de indivíduos cuja ação geralmente está relacionada a um sentimento de impossibilidade na identificação de alternativas viáveis para a solução de seus conflitos e sofrimentos, optando pelas tentativas de tirar a própria vida, como resposta.

Gráfico 2- Lesões autoprovocadas por adolescentes em Pernambuco, segundo sexo, no período de 2013-2017.

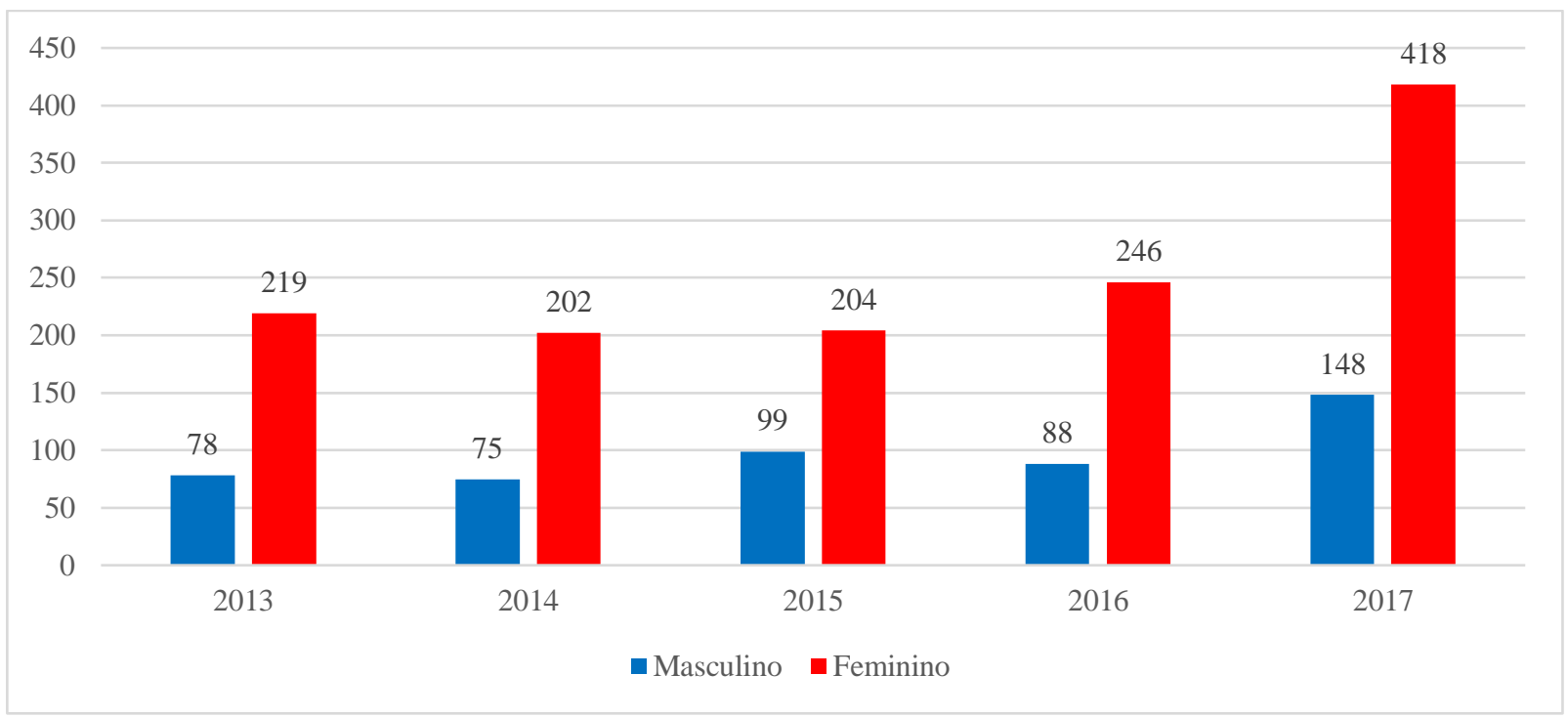

Fonte: Sistema de Informações de Agravos de Notificação

Em relação a variável de raça/cor, a cor com maior número de casos foi a parda, sendo essa responsável por mais da metade dos casos notificados (1096) correspondendo a 61,68\%, seguida pela cor branca com $16,71 \%$. Evidenciou-se também que muitos dos casos tiveram esta variável ignorada ou deixada em branco com um total de 250 (14,07\%). A partir da portaria $\mathrm{N}^{\circ}$ 344/2017 do Ministério da Saúde, tornou-se obrigatório o preenchimento deste quesito, respeitando sempre como o indivíduo se autodeclara e foi neste ano que houve uma redução 
dos casos deixados em branco. A cor preta correspondeu a 7,15\%, amarela a 0,64\% e indígena a $0,43 \%$ (Tabela 2 ).

Tabela 2- Lesões autoprovocadas por adolescentes em Pernambuco, segundo raça/cor, no período de 2013-2017.

\begin{tabular}{lcccccccccccc}
\hline Raça & $\mathbf{2 0 1 3}$ & $\mathbf{\%}$ & $\mathbf{2 0 1 4}$ & $\mathbf{\%}$ & $\mathbf{2 0 1 5}$ & $\mathbf{\%}$ & $\mathbf{2 0 1 6}$ & $\mathbf{\%}$ & $\mathbf{2 0 1 7}$ & $\mathbf{\%}$ & Total & \% \\
\hline $\begin{array}{l}\text { Ign/ } \\
\text { Branco }\end{array}$ & 57 & $19 \%$ & 52 & $19 \%$ & 57 & $19 \%$ & 49 & $15 \%$ & 35 & $6 \%$ & 250 & $14 \%$ \\
\hline Branca & 57 & $19 \%$ & 58 & $21 \%$ & 49 & $16 \%$ & 40 & $12 \%$ & 93 & $16 \%$ & 297 & $17 \%$ \\
\hline Preta & 18 & $6 \%$ & 20 & $7 \%$ & 18 & $6 \%$ & 20 & $6 \%$ & 31 & $5 \%$ & 107 & $6 \%$ \\
\hline Amarela & 0 & $0 \%$ & 1 & $0 \%$ & 4 & $1 \%$ & 2 & $1 \%$ & 4 & $1 \%$ & 11 & $1 \%$ \\
\hline Parda & 161 & $54 \%$ & 146 & $53 \%$ & 171 & $56 \%$ & 222 & $66 \%$ & 396 & $70 \%$ & 1096 & $62 \%$ \\
\hline Indígena & 4 & $1 \%$ & 0 & $0 \%$ & 4 & $1 \%$ & 1 & $0 \%$ & 7 & $1 \%$ & 16 & $1 \%$ \\
\hline Total & 297 & $100 \%$ & 277 & $100 \%$ & 303 & $100 \%$ & 334 & $100 \%$ & 566 & $100 \%$ & 1777 & $100 \%$ \\
\hline
\end{tabular}

Fonte: Sistema de Informações de Agravos de Notificação

Dessa forma, foi possível visualizar que houve um mal preenchimento do quesito raça/cor dentro do Sistema de Informação em Saúde (SIS), e esse mal preenchimento começa a ficar mais significativo quando se tornou a ficar obrigatório a partir da portaria $\mathrm{N}^{\circ} 344 / 2017$. Para Azevedo (2000), qualquer pessoa, com base nas inúmeras classificações que circulam, tanto oriundas do senso comum quanto do conhecimento transmitido academicamente, reconhece um negro, um branco ou um indígena. Cada um carrega uma construção da ideia de raça no seu imaginário. Porém, mesmo com a auto declaração esses sistemas ainda possuem uma escassez de dados e um preenchimento de forma errônea, o que faz com que o sistema não apresente um dado fidedigno da realidade.

Santos, Coelho e Araújo (2013) afirmam após resultados de uma pesquisa que muitos profissionais de saúde relatam que não é relevante declarar a cor do morto, porque já "se sabe de antemão" que "os pretos morrem mais", e morrem mais porque são pobres. São negros e pobres, mas, na opinião dos profissionais, só estavam lá porque eram pobres. A raça ou cor passa como algo acidental. Diante disso, percebe-se o quanto os casos são subnotificados e mal preenchidos nesse quesito, o que faz com que o sistema de informação de saúde passe a estar 
prestando informações errôneas devido ao seu mal preenchimento ou a subnotificação dos casos.

Por conseguinte, é importante pautar que a violência autoprovocada pela raça/cor é algo que cresce cada vez mais e que pode estar relacionado aos preconceitos arraigados culturalmente dentro do corpo social, que carrega o preconceito por muitos anos e o evidencia cada vez mais. Para Santos, Coelho, Araujo (2013), essa realidade é tão luminosa que chega a cegar, os negros morrem mais de forma indigna, matam-se e são mortos em proporções inusitadas e isso não causa nenhuma estranheza aos profissionais, à instituição e a sociedade.

\section{Conclusões}

O presente estudo identificou um cenário que fomenta a necessidade da criação de ações de políticas e programas preventivos direcionados a essa problemática no campo das políticas públicas de saúde direcionada a adolescentes e ao contexto da violência auto infligida. Faz-se urgente trabalhar sobre esse tema com a população de jovens para que se modifique prontamente esta situação no país, e criem-se condições para que este público procure atendimento nos serviços de saúde para que tenham suas necessidades atendidas e por isto, é imprescindível capacitar os profissionais, a fim de evitar desfechos negativos desta prática.

Além disso, é necessário romper com o tabu estabelecido acerca deste tema. Deve-se falar sobre isso com a sociedade bem como com a família, para que se evite o agravamento desta situação e se amenize este indicador. Ao se propagar este assunto, a população e os familiares estarão atentos a possíveis sinais que possam aparecer em parentes, amigos e conhecidos, e assim, poderão auxiliar na intervenção destes casos e evitar a culminância negativa destas práticas.

Por fim, conhecer a atual situação dos casos de lesões autoprovocadas poderá subsidiar a tomada de decisões por parte dos gestores para implementação e efetivação de políticas públicas voltadas ao assunto. Com o intuito de fortalecer a atenção à saúde destes indivíduos e serem ofertados serviços voltados ao cuidado destes de forma integral e equânime no âmbito do Sistema Único de Saúde.

\section{Referências}

Aguiar, C. R.; Carvalho, M. de O. G. BOLETIM EPIDEMIOLÓGICO 001/2018: LESÕES AUTOPROVOCADAS E SUICÍDIOS. SECRETARIA DE ESTADO DE SAÚDE DO 
RIODE JANEIRO, 2018.

Alves, M. A. G.; Cadete, M. M. M. Tentativa de suicídio infanto-juvenil: lesão da parte ou do todo?. Ciência \& Saúde Coletiva, 20(1):75-84, 2015.

AZEVEDO, E. Raça: conceito e preconceito. 2.ed. São Paulo: Ática, 1990.

BAHIA, Camila Alves et al. Lesão autoprovocada em todos os ciclos da vida: perfil das vítimas em serviços de urgência e emergência de capitais do Brasil. Ciênc. saúde coletiva, Rio de Janeiro, v. 22, n. 9, p. 2841-2850, Set. 2017.

Ballard ED, Cwik M, Storr CL, Goldstein M, Eaton WW, Wilcox HC. Recent medical service utilization and health conditions associated with a history of suicide attempts. General Hospital Psychiatry 2014; 36(4):437-441

BARBOSA, Fabiana de Oliveira; MACEDO, Paula Costa Mosca; SILVEIRA, Rosa Maria Carvalho da. Depressão e o suicídio. Rev. SBPH, Rio de Janeiro, v. 14, n. 1, p. 233-243, jun. 2011.

BOTEGA NJ. Prática no Hospital Geral: interconsulta e emergência. Porto Alegre: Artmed; 2002.

BRASIL, Secretaria de Vigilância em Saúde, Ministério da Saúde. Boletim Epidemiológico: Perfil epidemiológico das tentativas e óbitos por suicídio no Brasil e a rede de atenção à saúde. Volume $48, \mathrm{~N}^{\circ} 30-2017$.

Cicogna, J. I. R.; Hillesheim, D.; Hallal, A. L. de L. C. Mortalidade por suicídio de adolescentes no Brasil: tendência temporal de crescimento entre 2000 e 2015. J. bras. psiquiatr. vol.68 no.1 Rio de Janeiro, 2019.

Freud S. Introdução ao narcisismo: ensaios de metapsicologia e outros textos [1914-1916]. São Paulo: Schwarcz; 2010. (Coleção Obras Completas, v.12)

HESKETH, J. L.; CASTRO, A. G. de. Fatores correlacionados com a tentativa de suicídio. Rev. Saúde Pública, S. Paulo, v.12, p.138-146, 1978.

MACHIN, R.. NEM DOENTE, NEM VÍTIMA: O ATENDIMENTO ÀS "LESÕES AUTOPROVOCADAS” NAS EMERGÊNCIAS. Cien Saude Colet, Dez., 2007.

MELEIRO A, Teng CT, Wang YP. Suicídio: estudos fundamentais. São Paulo: Segmento Farma; 2004.

MONTEIRO, Rosane Aparecida et al. Hospitalizações relacionadas a lesões autoprovocadas intencionalmente - Brasil, 2002 a 2013. Ciênc. saúde coletiva, Rio de Janeiro, v. 20, n. 3, p. 689-699, Mar. 2015.

Organização Mundial da Saúde (OMS). Prevenção do suicídio: um imperativo global. Genebra: OMS; 2014. 
Organização Mundial da Saúde. Prevenção do Suicido: um manual para profissionais da saúde em atenção primária. Genebra: 2000.

RIBEIRO, José Mendes; MOREIRA, Marcelo Rasga. Uma abordagem sobre o suicídio de adolescentes e jovens no Brasil. Ciênc. saúde coletiva, Rio de Janeiro, v. 23, n. 9, p. 28212834, set. 2018.

SANTOS, Andreia Beatriz Silva dos; COELHO, Thereza Christina Bahia; ARAUJO, Edna Maria de. Identificação racial e a produção da informação em saúde. Interface (Botucatu), Botucatu, v. 17, n. 45, p. 341-356, Jun. 2013.

TEIXEIRA, Ana Maria Fortaleza; LUIS, Margarita Antonia Villar. Suicídio, lesões e envenenamento em adolescentes: um estudo epidemiológico. Rev. Latino-Am. Enfermagem, Ribeirão Preto, v. 5, n. spe, p. 31-36, 1997. 